\title{
Perceptions about the aetilogy, treatment and prevention of convulsions in under-five children in Rumphi.
}

\section{Munthali AC}

\section{Summary}

This paper, based on an ethnographic study carried out in western Rumphi, examines people's perceptions about the aetiology, treatment and prevention of convulsions in underfive children. Both old and young women attributed convulsions to the eating of eggs by under-five children and acts of witchcraft. However, young women, probably because of their higher level of educational attainment and their attendance of the health education sessions conducted by health surveillance assistants, also interpreted convulsions as serious forms of malaria. As regards prevention of convulsions, informants mentioned traditional methods such as the use of incisions and wearing of amulets, and that children should not eat eggs. Because of the attribution of convulsions to witcheraft and such other culturally related-causes, most informants said that treatment for this illness is first sought from traditional healers, and biomedicine is only sought when the situation worsens. The delay in seeking therapy for convulsing children from modern medical facilities (mainly because of culturally perceived causes and modes of prevention) diminishes chances of the survival of such children. The knowledge of such community perceptions about convulsions is necessary as it can better inform the design and implementation of health education programmes.

\section{Introduction}

Malaria is a huge public health problem in Malawi. In 1996, there were 2,628,539 first visits to the health facilities that were diagnosed as malaria ${ }^{1}$. In 2000 , malaria was responsible for 43 percent of all outpatient cases and 19 percent of under-five mortality ${ }^{2}$. While adults also suffer from malaria, pregnant women and under-five children are the ones who are most vulnerable. While the occurrence of convulsions, splenomegaly and anaemia in under-five children can be attributed to other causes, but in malaria endemic areas, such as in Malawi, these conditions are mostly due malaria.

This paper examines what people in western Rumphi perceive to be the aetiology of convulsions in under-five children and the methods that they use to prevent this illness. The paper further discusses the therapy seeking process that mothers pursue when under-five children suffer from convulsions. Research upon which this paper is based was done between May 2000 and May 2002.

\footnotetext{
Materials and Methods

This was a qualitative study and participant observation, indepth interviews with old men and women and mothers with under-five children and key informant interviews with traditional healers, traditional birth attendants, health surveillance assistants and medical assistants comprised the major data collection methods.
}

\section{Results}

Perceptions about the aetiology and prevention of convulsions

During focus group discussions and in-depth interviews, a number of causative factors for convulsions in under-five children emerged of which the consumption of eggs by these children was the major one. The belief that children will suffer from convulsions when they eat eggs seems to be widespread. This has also been reported to occur amongst the Tonga of Nkhata Bay and the Ngoni of Mzimba ${ }^{3}$. This is however in conflict with biomedicine which promotes that children should eat eggs as they are an important source of protein. In addition to eating eggs, some informants also said that witchcraft can also cause convulsions in under-five children. There are a number of ways through which people know that convulsions are due to witchcraft. As we shall discuss below, there exist among the Tumbuka specific traditional medicines which are seen as efficacious for the treatment of convulsions. The failure of such medicines to cure convulsions leads people to suspect or conclude that witcheraft is involved. Diviners can also be consulted who, as far as the Tumbuka are concerned, are able to establish that the illness is due to witchcraft and they can also administer appropriate traditional medicines.

While both young and old informants mostly attributed convulsions in under-five children to the consumption of eggs and acts of witcheraft, few young women with under-five children however linked convulsions to malaria. These women said that they had learnt this during under-five clinics as well as from their friends and relatives. None of the old men and women (who were aged above 65 years) ever said that convulsions are an indication of severe malaria. They looked at convulsions as a separate disease entity. Mothers, in general, considered convulsions to be very dangerous and said that if proper treatment is not sought, the child might die, go mad or the disease might develop into epilepsy when the child grows old.

As regards prevention, most women mentioned that children should not eat eggs. Informants also mentioned other indigenous preventative methods, and these included the making of incisions on the child's body and rubbing some medicines into these cuts, children wearing amulets made from some part of a warthog, and the use of strong smelling plants.

\section{Seeking therapy for convulsions in under-five children}

Most of the informants said that whenever a child suffers from convulsions, they first consult traditional healers or knowledgeable old men and women for traditional medicine. Only one traditional healer said that since a child suffering from convulsions runs a very high fever, she first covered the convulsing child with a clean wet cloth in order to bring down the body temperature before administering traditional medicines.

The traditional medicines used to treat convulsions mostly consist of strong smelling plants. In western Rumphi, the most popular herb used for the treatment of convulsions in under-five children is kanufu. Traditional healers said that strong smelling plants are used because they believe that the smell chases the convulsions away. Leaves from these strong smelling plants are rubbed between the palms and then put very close to the nose of the child so that he or she should inhale the smell. These leaves are also rubbed all over the child's body, including the head. According to informants, when these strong smelling plant species are administered, the major indicator that the child will be or has been cured is that he or she defecates and urinates. If the child does not do this, then he or she is forced to do so through enema. The use of strong smelling herbs has also been 
reported in other parts of Malawi, for example in Nkhata Bay and Kasungu, the plants kamununkha and mphungabwi are used respectively ${ }^{3}$. People expect that these traditional medicines for the treatment of convulsions will work unless there is witchcraft involved. Traditional healers said that some of the traditional medicines for convulsions may not work because the child may possibly be suffering from epilepsy and not convulsions, as some people might not differentiate between the two. Hence, instead of looking for treatment for epilepsy, they look for treatment for convulsions and the child cannot get cured. They however said that a child with epilepsy produces a lot of foam at the mouth while the one with convulsions is accompanied by fever. If the child does not recover after being given strong smelling traditional medicines, he or she is then taken to the local health centre for treatment. While this is the case, informants said that some women tend to get worried and even start crying when a child suffering from convulsions is taken to the hospital. They cry because they anticipate that the child will not survive as they have learnt from experience that there are no medicines for convulsions at the health centre. They further claimed that the treatment that they receive at the hospital (which in most cases consists of an injection) actually worsens the condition of the child, eventually killing him.

Despite the fact that traditional forms of treatment for convulsions are widely used, there were others who felt that it was a waste of time to use these forms of treatment; they have leant this from experience ad they simply take their children to the health centres for treatment.

\section{Discussion}

Children suffering from convulsions are first treated using traditional medicine. Such children are only brought to the health centre very late when the disease is in its advanced stages and the chances of survival for such children might be very slim. These children are given an injection and when they die, people generally attribute the death to the injection, and not to the fact that the child was brought to the health centre late. During the study, it seemed that people do not perceive that the delay in seeking appropriate care when children suffers from convulsions is detrimental to the children's health; hence when children die after being given an injection, they blame the hospital staff of killing their children and do not acknowledge that they only went to health centre when it was too late.

Despite the health education that is given during the under-five clinics, most young women did not link convulsions with malaria. These people claimed that Fansidar SP cures a child with malaria while most of the children with convulsions do not survive even after being given a dosage of Fansidar SP. They make a conclusion that since Fansidar cannot cure convulsions and the drug is the right medication for malaria, then convulsions are not related to malaria. There were a few women however who linked convulsions to malaria and such women said that as soon as a child starts convulsing, they go with him or her to the health centre for treatment. However, even these women first administer some traditional medicines before going to the health centre for treatment. For such women, "the use of traditional medicine supplements rather than replaces modern health care ${ }^{4}$ i.e. they perceive traditional medicine as relieving the symptoms but not necessarily curing. The few women who recognize that convulsions are linked to malaria and their positive experiences at the health centers may well act as agents of change in society and therefore bring about change to allopathic views of convulsions on the part of their fellow villagers.

\section{Conclusion}

While some women indeed go with their children to health centers when they suffer from convulsions, some do not go because of the belief that there is no appropriate treatment for such an illness at the health centers. Others are forced to go to health centres when the situation worsens. The delays in seeking hospital treatment for convulsions reduce the likelihood of children being cured. Ethnographic studies such as this one are useful in that they spell out the communities' perceptions about the causes and methods of prevention of convulsions and how these can influence the therapy seeking process when a child suffers from this illness. Such results are of practical importance to Malawi as they can be used to better inform the public health education programmes.

Munthali AC

Centre for Social Research, University of Malawi, Zomba

P.O. Box 278

Zomba

Email:munthali65@yahoo.com

References

1. Ministry of Health and Population-HMIS. Basic health statistics 1996-report 20. Lilongwe, Capital Printing Press, 1999.

2. National Statistical Office. Demographic and Health Survey. Zomba: National Statistical Office, 2001

3. Matinga PU and Munthali. A. An ethnographic study on malaria in five selected districts in Malawi (Final report submitted to UNICEF Malawi, Lilongwe), 2001

4. Baume C, Helitzer D and Kachur SP. Patterns of care for childhood malaria in Zambia. Social Science and Medicine 2000, 25:277-292.

\section{T.O.W TAFATATHA ORTHOPEADIC WORKS}

Tafatatha Orthopeadic Works is a Private Workshop which was opened on the 13th October, 2000. It is the only private orthopeadIc workshop in the entire country which uses modern orthopeadic technologies. The Workshop is situated in Newlands. It is run by a qualified Orthopeadic Technologist.

High quality orthoses which are made from thermoplastics can be obtained at T.O.W. We will soon also be making prostheses using the lamination method.

The following are some of the appliances which can be obtained at T.O.W:

- Cervical collars

- Knee braces

- Elbow crutches

- Shoe raise

- Clubfoot splints

- Ankle braces

- Finger splints

- Wrist splints

You can get in touch with us on the following detalls: Contact person : Wilie Tafatatha Address PO Box 90592 Bangwe Blantyre 9

Terephone cellphone .01 .644194

E-mail

- tafatatha@malawinet 